\title{
Diagnostics of molecular orbital quality
}

\author{
Jia Deng, Andrew T.B. Gilbert, and Peter M.W. Gill
}

\begin{abstract}
We discuss several measures of the quality of a molecular orbital. Each requires only that the orbital be associated with a well-defined Fock operator and is thus applicable to both Hartree-Fock and density functional orbitals. One of the measures, the $\gamma$ diagnostic, ranges from $\gamma=0$ (perfect) to $\gamma=\pi / 2$ (poor) and is conceptually simple. We illustrate its usefulness by applying it to a number of small atoms and ions.
\end{abstract}

Key words: molecular orbital quality, density functional theory, Hartree-Fock.

Résumé : On discute de plusieurs mesures de la qualité d'une orbitale moléculaire. Chacune nécessite uniquement que l'orbitale soit associée à un opérateur de Fock bien défini et qu'il soit ainsi applicable aux orbitales de Hartree-Fock ainsi qu'aux fonctionnelles de densité. L'une ce ces mesures, le diagnostic, s'étend de 0 (parfait) à 2 (mauvais) et elle est conceptuellement simple. On illustre son utilité en l'appliquant à un certain nombre de petits atomes et de petits ions.

Mots-clés : qualité d'une orbitale moléculaire, théorie de la fonctionnelle de la densité, Hartree-Fock.

[Traduit par la Rédaction]

\section{Introduction}

Some say that quantum chemistry is a branch of applied mathematics and that, by implication, quantum chemists are applied mathematicians. One can certainly mount a strong argument in favor of this. After all, quantum chemistry is primarily concerned with finding approximate solutions to a certain linear second-order partial differential equation and using those solutions to comprehend the chemical behavior of matter. However, if we are indeed applied mathematicians, we break one of the cardinal rules of our order: with few exceptions, we never check how well our "solutions" satisfy the equation!

How well does a given wave function satisfy the Schrödinger equation? At the conclusion of a quantum chemical calculation, any self-respecting software package ought to address this question and provide a quantitative answer that the user can interpret as a quality measure. However, it turns out that this raises a number of interesting mathematical issues, and we have therefore sought initially to address the more modest question: How well does a given orbital satisfy its self consistent field (SCF) equation?

Standard practice in quantum chemistry is to gauge the quality of a molecular orbital by comparing it with the orbital obtained from a calculation with a larger basis set. However, this approach is both expensive and non-rigorous, for it is possible that the larger basis may suffer from the same deficiencies as the smaller one. Moreover, it is aesthetically unsatisfactory to judge an orbital by comparing it with another: it ought to be possible to appraise it purely on its own merits. In this paper, we propose a method for assessing the quality of a finite-basis SCF orbital in this way, without the need to perform a larger-basis calculation.
Although our approach is applicable to any orbital that is associated with a well-defined Fock operator, we will confine our numerical tests here to Hartree-Fock orbitals. The extension to density functional theory (DFT) orbitals is conceptually straightforward but requires consideration of DFT quadrature grids. We use atomic units throughout.

\section{Molecular orbital diagnostics}

An exact orbital is an eigenfunction of its Fock operator. ${ }^{1}$ However, an orbital $\psi$ from a perfectly converged finite-basis Hartree-Fock ${ }^{2-4}$ or Kohn-Sham ${ }^{5}$ calculation is not an eigenfunction of its own Fock operator $\boldsymbol{F}$, that is,

$$
\text { [1] } \quad \boldsymbol{F} \psi \neq \varepsilon \psi
$$

unless the basis happens to span the exact orbital. ${ }^{6}$ This under-appreciated fact holds the key to our approach because the difference between the two sides of eq. [1] measures the quality of the orbital. There are several ways to exploit this. One approach is based on the local orbital energy

$$
\text { [2] } \quad \epsilon(\mathbf{r})=(\boldsymbol{F} \psi) / \psi
$$

If the orbital were perfect, $\epsilon(\mathbf{r})$ would be constant everywhere; small imperfections in $\psi$ will produce small variations in $\epsilon(\mathbf{r})$.

A second approach recognizes that the normalized versions of $\psi$ and $\boldsymbol{F} \psi$ should be identical. Accordingly, the discrepancy

$$
\text { [3] } \delta(\mathbf{r})=\frac{\psi}{\sqrt{\langle\psi \mid \psi\rangle}}-\frac{\boldsymbol{F} \psi}{\sqrt{\langle\boldsymbol{F} \psi \mid \boldsymbol{F} \psi\rangle}}
$$

Received 31 December 2009. Accepted 18 February 2010. Published on the NRC Research Press Web site at canjchem.nrc.ca on 13 May 2010 .

J. Deng, A.T.B. Gilbert, and P.M.W. Gill. ${ }^{1}$ Research School of Chemistry, Australian National University, ACT 0200, Australia.

${ }^{1}$ Corresponding author (e-mail: peter.gill@anu.edu.au). 
Fig. 1. The $\gamma$ diagnostic for the orbital $\exp (-\alpha r)$ in the $\mathrm{H}$ atom.

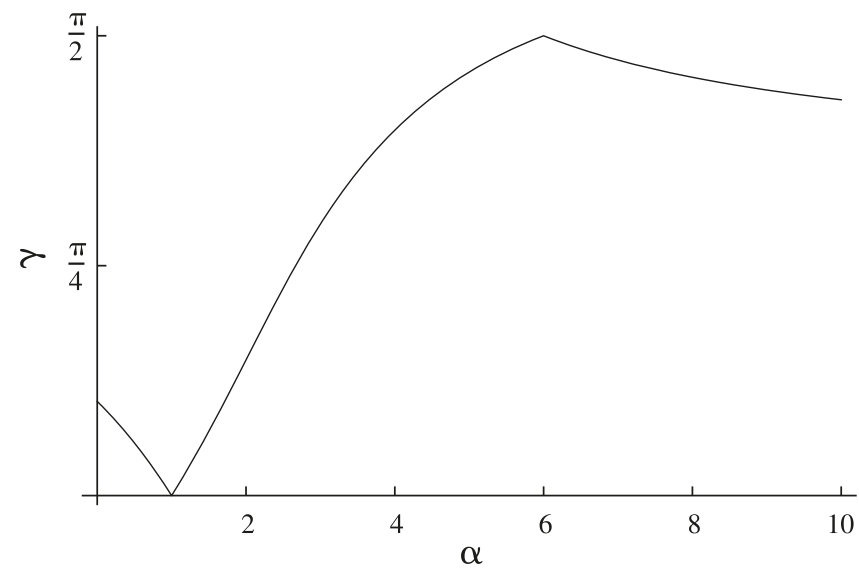

Table 1. $\gamma$ values for Kellner orbitals in the He-like ions.

\begin{tabular}{llllllll}
\hline & $\mathrm{H}^{-}$ & $\mathrm{He}$ & $\mathrm{Li}^{+}$ & $\mathrm{Be}^{2+}$ & $\mathrm{B}^{3+}$ & $\mathrm{C}^{4+}$ & $\mathrm{N}^{5+}$ \\
\hline$Z$ & 1 & 2 & 3 & 4 & 5 & 6 & 7 \\
$\gamma$ & 0.488 & 0.063 & 0.033 & 0.023 & 0.017 & 0.014 & 0.012 \\
\hline
\end{tabular}

should vanish at all points $\mathbf{r}$ and, wherever it fails to do so, it reveals deficiencies in the orbital.

A third approach determines the angle

$$
\text { [4] } \begin{aligned}
\gamma & =\cos ^{-1} \sqrt{\frac{\langle\psi \mid \boldsymbol{F} \psi\rangle^{2}}{\langle\psi \mid \psi\rangle\langle\boldsymbol{F} \psi \mid \boldsymbol{F} \psi\rangle}} \\
& =\tan ^{-1} \sqrt{\frac{\langle\psi \mid \psi\rangle\langle\boldsymbol{F} \psi \mid \boldsymbol{F} \psi\rangle}{\langle\psi \mid \boldsymbol{F} \psi\rangle^{2}}-1}
\end{aligned}
$$

between $\psi$ and $\boldsymbol{F} \psi$. Unlike $\epsilon(\mathbf{r})$ and $\delta(\mathbf{r})$, which are functions, $\gamma$ is a number between 0 (perfect) and $\pi / 2$ (poor) and achieves the former value if, and only if, $\psi$ is an eigenfunction of $\boldsymbol{F}$.

To calculate numerical values of $\gamma$, we must choose a suitable inner product $\langle\cdot \mid \cdot\rangle$. The overlap metric

$$
\text { [5] }\langle f \mid g\rangle=\int f(\mathbf{r}) g(\mathbf{r}) \mathrm{d} \mathbf{r}
$$

is an obvious choice but it yields poor $\gamma$ values if the orbital fails to possess the correct nuclear cusps. This is a particularly serious problem if the orbital is expanded in a nuclearcentered Gaussian basis because, close to a nucleus, any Gaussian is flat and consequently $\boldsymbol{F} \psi \sim(-Z / r) \psi$ is singular.

Because of the widespread popularity of Gaussian basis sets, we prefer an inner product that is less sensitive to such cusp violations. We have found that the Coulomb metric

$$
\text { [6] }\langle f \mid g\rangle=\iint \frac{f\left(\mathbf{r}_{1}\right) g\left(\mathbf{r}_{2}\right)}{r_{12}} \mathrm{~d} \mathbf{r}_{1} \mathrm{~d} \mathbf{r}_{2}
$$

works well and we have adopted it throughout this work. If $\psi$ is a sum of Gaussians, all of the inner products needed for atoms can be evaluated in terms of elementary functions, using integrals such as

$$
\text { [7] }\left\langle\frac{\mathrm{e}^{-b^{2} r^{2}}}{r} \mid \frac{\mathrm{e}^{-d^{2} r^{2}}}{r}\right\rangle=4 \pi^{5 / 2} \frac{b+d-\sqrt{b^{2}+d^{2}}}{b^{2} d^{2}}
$$

A table of such integrals is given in the Appendix.

In this preliminary exploration, we will investigate the performance of the $\gamma$ diagnostic (eq. [4]) in very small systems. The primary question is whether or not $\gamma$ behaves "reasonably".

\section{Examples}

\section{Hydrogen atom}

This one-electron system has the Fock operator

$$
\text { [8] } \quad \boldsymbol{F}=-\frac{\nabla^{2}}{2}-\frac{1}{r}
$$

and its true orbital is $\exp (-r)$. To begin our exploration of the $\gamma$ diagnostic, we consider an HF calculation using the single basis function $\exp (-\alpha r)$. The computed orbital will differ from the true orbital except, of course, when $\alpha=1$.

The $\gamma$ diagnostic (eq. [4]), using the Coulomb metric (eq. [6]), is

$$
\text { [9] } \quad \gamma=\tan ^{-1}\left|2 \frac{\alpha-1}{\alpha-6}\right|
$$

and this is plotted, for $0<\alpha<10$, in Fig. 1. As expected, $\gamma$ vanishes for $\alpha=1$ and grows almost linearly as $\alpha$ deviates from this optimal value. For $\alpha=6$, it turns out that $\psi$ and $\boldsymbol{F} \psi$ are exactly orthogonal, i.e., the angle between them is $\pi / 2$.

\section{Helium-like ions}

These two-electron systems have the Fock operator

$$
\boldsymbol{F}=-\frac{\nabla^{2}}{2}-\frac{Z}{r}+\int \frac{\psi\left(\mathbf{r}^{\prime}\right)^{2}}{\left|\mathbf{r}-\mathbf{r}^{\prime}\right|} \mathrm{d} \mathbf{r}^{\prime}
$$

where $Z$ is the nuclear charge. The true HF orbital is unknown, but if we approximate it by the Kellner orbital ${ }^{7}$

$$
\text { [11] } \quad \psi(\mathbf{r})=\exp (-\alpha r)
$$

the HF energy is minimized by choosing $\alpha=Z-5 / 16$.

How satisfactory is this orbital? It can be shown that

$$
\text { [12] } \quad \gamma=\tan ^{-1}\left|\frac{a}{Z-b}\right|
$$

where $a=\sqrt{1987 / 388800}$ and $b=623 / 720$ and this is evaluated in Table 1 for $Z=1, \ldots, 7$. These results reveal that the Kellner orbital is a good approximation $(\gamma \approx 0.01)$ to the true orbital for large- $Z$ ions, such as $\mathrm{C}^{4+}$ and $\mathrm{N}^{5+}$, that it is reasonable $(\gamma \approx 0.05)$ for the He atom itself, but that it is very poor $(\gamma \approx 0.5)$ for the $\mathrm{H}^{-}$ion. This may be related to the difficulties that Cioslowski and others have encountered when seeking an accurate HF orbital for the hydride anion. 8,9

\section{Hookium atom}

This two-electron system ${ }^{10,11}$ has the Fock operator

$$
\boldsymbol{F}=-\frac{\nabla^{2}}{2}+\frac{r^{2}}{8}+\int \frac{\psi\left(\mathbf{r}^{\prime}\right)^{2}}{\left|\mathbf{r}-\mathbf{r}^{\prime}\right|} \mathrm{d} \mathbf{r}^{\prime}
$$

and its true HF orbital is unknown. However, neglecting the 
Table 2. $\gamma$ values for Gaussian and Ragot orbitals in hookium.

\begin{tabular}{llllll}
\hline Orbital & Equation & $\alpha$ & $\beta$ & $\gamma$ \\
\hline Gaussian & {$[14]$} & 0.25 & - & & 0.0430 \\
Gaussian & {$[14]$} & 0.210549021 & - & & 0.0142 \\
Ragot & {$[15]$} & 0.25 & 2.771219931 & 0.0007 \\
Ragot & {$[15]$} & 0.251117376 & 2.711087898 & 0.0002 \\
\hline
\end{tabular}

Table 3. $\gamma$ values for pc- $n$ and aug-pc- $n$ orbitals in small atoms and ions.

\begin{tabular}{|c|c|c|c|c|c|c|c|c|c|c|c|}
\hline & \multirow[b]{2}{*}{ Orbital } & \multicolumn{5}{|l|}{ pc- $n$} & \multicolumn{5}{|c|}{ aug-pc- $n$} \\
\hline & & 0 & 1 & 2 & 3 & 4 & 0 & 1 & 2 & 3 & 4 \\
\hline \multirow[t]{2}{*}{$\mathrm{H}$} & $1 \mathrm{~s}$ & 0.0436 & 0.0255 & 0.0079 & 0.0020 & 0.0007 & 0.0403 & 0.0313 & 0.0085 & 0.0015 & 0.0005 \\
\hline & $2 \mathrm{~s}$ & 0.7425 & 0.7202 & 0.7131 & 0.7323 & 0.7449 & 0.7491 & 0.7573 & 0.7735 & 0.7917 & 0.8007 \\
\hline \multirow[t]{2}{*}{$\mathrm{He}^{+}$} & $1 \mathrm{~s}$ & 0.0650 & 0.0443 & 0.0133 & 0.0035 & 0.0017 & 0.0811 & 0.0495 & 0.0135 & 0.0037 & 0.0016 \\
\hline & $2 \mathrm{~s}$ & 1.4198 & 1.3296 & 0.6133 & 0.3130 & 0.2424 & 0.1309 & 0.1110 & 0.0839 & 0.0563 & 0.0445 \\
\hline $\mathrm{H}^{-}$ & $1 \mathrm{~s}$ & 0.9572 & 0.7502 & 0.4354 & 0.2507 & 0.1873 & 0.1363 & 0.0870 & 0.0554 & 0.0348 & 0.0267 \\
\hline $\mathrm{He}$ & $1 \mathrm{~s}$ & 0.0756 & 0.0389 & 0.0110 & 0.0022 & 0.0010 & 0.0620 & 0.0396 & 0.0125 & 0.0022 & 0.0009 \\
\hline $\mathrm{Li}^{+}$ & $1 \mathrm{~s}$ & 0.0297 & 0.0151 & 0.0119 & 0.0122 & 0.0042 & 0.0295 & 0.0152 & 0.0109 & 0.0124 & 0.0042 \\
\hline \multirow[t]{2}{*}{$\mathrm{Be}$} & $1 \mathrm{~s}$ & 0.0349 & 0.0153 & 0.0082 & 0.0091 & 0.0034 & 0.0339 & 0.0150 & 0.0079 & 0.0093 & 0.0034 \\
\hline & $2 s$ & 0.0881 & 0.0203 & 0.0123 & 0.0029 & 0.0031 & 0.0358 & 0.0147 & 0.0152 & 0.0029 & 0.0030 \\
\hline
\end{tabular}

electron repulsion yields the harmonic oscillator wave function $\exp \left(-r^{2} / 4\right)$ and one may therefore anticipate that

[14] $\quad \psi(\mathbf{r})=\exp \left(-\alpha r^{2}\right)$

may be a good guess. The HF energy of this orbital, using an optimized exponent, lies $1.5 \mathrm{~m} E_{\mathrm{h}}$ above the HF limit. However, Ragot has recently claimed ${ }^{12}$ that the modified orbital

$$
[15] \quad \psi(\mathbf{r})=\sqrt{r^{2}+\beta^{2}} \exp \left(-\alpha r^{2}\right)
$$

is very much better, provided that $\alpha$ and $\beta$ are chosen carefully, and constructs two orbitals of this form, lying 4.5 and $0.6 \mu E_{\mathrm{h}}$ above the HF limit, respectively.

The $\gamma$ diagnostic allows us to compare the quality of these orbitals, and though we have not been able to find $\gamma$ in closed form for eq. [15] the required integrals can be found by quadrature. The results shown in Table 2 reveal that the Gaussian (eq. [14]) is a good approximation to the exact orbital but that the Ragot orbitals (eq. [15]) are superior by one to two orders of magnitude.

\section{Other small systems}

Table 3 lists $\gamma$ values for the orbitals in some small atoms and ions, using Jensen's pc- $n$ basis sets. ${ }^{13,14}$

$\mathrm{H}$ and $\mathrm{He}^{+}$ions are one-electron species whose 1s orbitals are modeled well by the pc- $n$ bases. It is not surprising that the $\mathrm{He}^{+}$orbital is slightly inferior to that of $\mathrm{H}$, for the helium bases were designed to be optimal for the neutral atom, not the much more compact cation. Nevertheless, the triple-zeta pc- 2 orbitals are good and the pc-3 and pc-4 orbitals are excellent.

The 2 s orbitals (the LUMOs in these species) tell a very different story. In the $\mathrm{H}$ atom, the $\gamma$ values are appalling, and actually deteriorate as the basis set quality improves. This arises because, in the HF picture, the virtual orbitals of the $\mathrm{H}$ atom experience an exponentially decaying attractive potential and therefore describe essentially free electrons.
Of course, nuclear-centered Gaussian basis sets (e.g., augpc- $n$ ) cannot capture such behavior. The situation is better in $\mathrm{He}^{+}$because the virtual orbitals experience a Coulombic attractive potential and, consequently, approximate the standard hydrogenic orbitals. Although they are too diffuse to be modelled well by pc- 0 , pc- 1 , and $\mathrm{pc}-2$, the higher aug-pc- $n$ bases do a better job.

$\mathrm{H}^{-}, \mathrm{He}$, and $\mathrm{Li}^{+}$are isoelectronic but it is clear that their pc- $n$ 1s orbitals vary greatly in quality. While good orbitals are provided by pc-1 (for $\mathrm{Li}^{+}$), and pc-2 (for $\mathrm{He}$ ), a satisfactory orbital for the hydride ion is not obtained even with the septuple-zeta pc-4 basis. This arises because the second electron in $\mathrm{H}^{-}$is barely bound, and diffuse functions therefore play a crucial role in the basis. Accordingly, we find that the aug-pc- $n$ orbital is consistently almost an order of magnitude better than its pc- $n$ analog; indeed, even the lowly aug-pc- 0 orbital exceeds the quality of the pc- 4 orbital.

The last two lines in Table 3 pertain to the core and valence orbitals in the $\mathrm{Be}$ atom. We find that the $1 \mathrm{~s}$ orbital is accurately modelled, even by the small pc- $n$ bases, but that the $2 \mathrm{~s}$ orbital is more challenging and benefits significantly from the addition of diffuse functions. It is surprising to find that, in passing from the pc-2 to the pc-3 basis set, the valence orbital improves dramatically but the core orbital actually becomes slightly worse. This anomaly is unaffected by the addition of diffuse functions.

\section{Concluding remarks}

It is impossible to assess the quality of an SCF calculation from its energy alone, and as a result, quantum chemists have traditionally been compeled to perform sequences of calculations, looking for signs of convergence as the basis set is systematically improved. In contrast, the $\gamma$ diagnostic (eq. [4]) exploits the inequality (eq. [1]) to provide a chemist with a direct measure of the quality of a computed orbital.

Using the Coulomb metric (eq. [6]), it appears empirically 
that "reasonable" orbitals have $\gamma \leqq 0.05$, "good" orbitals have $\gamma \leqq 0.01$, and "excellent" orbitals have $\gamma \leqq 0.005$.

In this preliminary work, we have computed $\gamma$ for singlecenter systems only. To facilitate the efficient calculation of $\gamma$ for the MOs in polyatomic molecules, the integrals in the Appendix must be generalized to the multicenter case. In large systems, straightforward evaluation of the $\langle\boldsymbol{F} \psi \mid \boldsymbol{F} \psi\rangle$ integral becomes computationally costly, but techniques based on the resolution of the identity (RI) ${ }^{15-17}$ or resolution of the Coulomb operator ${ }^{18-20}$ should be helpful in this context.

In the case of Kohn-Sham orbitals, the inner products in eq. [4] involving $\boldsymbol{F} \psi$ cannot be computed in closed form, and one must resort instead to quadrature. We will discuss this elsewhere.

In this brief paper, we have proposed a diagnostic to quantify orbital quality and have studied its performance on a few very small atoms and ions. We will implement this in the Q-Chem package ${ }^{21}$ and will report molecular results elsewhere. We also plan to extend these ideas to a wave function diagnostic in the near future.

\section{Acknowledgement}

We thank Pierre-François Loos for making a number of helpful comments on this manuscript.

\section{References}

(1) Szabo, A.; Ostlund, N. S. Modern Quantum Chemistry; McGraw-Hill: New York, 1989.

(2) Roothaan, C. C. J. Rev. Mod. Phys. 1951, 23 (2), 69. doi:10. 1103/RevModPhys.23.69.

(3) Hall, G. G. Proc. R. Soc. Lond. 1951, A205, 541.

(4) Pople, J. A.; Nesbet, R. K. J. Chem. Phys. 1954, 22 (3), 571. doi:10.1063/1.1740120.

(5) Pople, J. A.; Gill, P. M. W.; Johnson, B. G. Chem. Phys. Lett. 1992, 199 (6), 557. doi:10.1016/0009-2614(92)85009Y.

(6) Deng, J.; Gilbert, A. T. B.; Gill, P. M. W. J. Chem. Phys. 2009, 130 (23),o. 231101. doi:10.1063/1.3152864.

(7) Kellner, G. W. Z. Phys. 1927, 44 (1-2), 91. doi:10.1007/ BF01391720.

(8) Cioslowski, J.; Liu, G. J. Chem. Phys. 1998, 109 (19), 8225. doi:10.1063/1.477484.

(9) Pearson, J. K.; Gill, P. M. W.; Ugalde, J.; Boyd, R. J. Mol. Phys. 2009, 107 (8), 1089. doi:10.1080/00268970902740563.

(10) Kais, S.; Herschbach, D. R.; Levine, R. D. J. Chem. Phys. 1989, 91 (12), 7791. doi:10.1063/1.457247.

(11) O'Neill, D. P.; Gill, P. M. W. Phys. Rev. A 2003, 68 (2),o. 022505. doi:10.1103/PhysRevA.68.022505.
(12) Ragot, S. J. Chem. Phys. 2008, 128 (16),o. 164104. doi:10. 1063/1.2904874.

(13) Jensen, F. J. Chem. Phys. 2001, 115 (20), 9113. doi:10.1063/ 1.1413524.

(14) Jensen, F. J. Phys. Chem. A 2007, 111 (44), 11198. doi:10. 1021/jp068677h. PMID:17439196.

(15) Baerends, E. J.; Ellis, D. E.; Ros, P. Chem. Phys. 1973, 2 (1), 41. doi:10.1016/0301-0104(73)80059-X.

(16) Dunlap, B. I.; Connolly, J.; Sabin, J. J. Chem. Phys. 1979, 71 (8), 3396. doi:10.1063/1.438728.

(17) Vahtras, O.; Almlöf, J.; Feyereisen, M. Chem. Phys. Lett. 1993, 213 (5-6), 514. doi:10.1016/0009-2614(93)89151-7.

(18) Varganov, S. A.; Gilbert, A. T. B.; Deplazes, E.; Gill, P. M. W. J. Chem. Phys. 2008, 128 (20),o. 201104. doi:10.1063/1. 2939239.

(19) Gill, P. M. W.; Gilbert, A. T. B. Chem. Phys. 2009, 356 (13), 86. doi:10.1016/j.chemphys.2008.10.047.

(20) Limpanuparb, T.; Gill, P. M. W. Phys. Chem. Chem. Phys. 2009, 11 (40), 9176. doi:10.1039/b910613h. PMID: 19812838.

(21) Shao, Y.; Molnar, L. F.; Jung, Y.; Kussmann, J.; Ochsenfeld, C.; Brown, S. T.; Gilbert, A. T. B.; Slipchenko, L. V.; Levchenko, S. V.; O’Neill, D. P.; DiStasio, R. A., Jr.; Lochan, R. C.; Wang, T.; Beran, G. J. O.; Besley, N. A.; Herbert, J. M.; Lin, C. Y.; Van Voorhis, T.; Chien, S. H.; Sodt, A.; Steele, R. P.; Rassolov, V. A.; Maslen, P. E.; Korambath, P. P.; Adamson, R. D.; Austin, B.; Baker, J.; Byrd, E. F. C.; Dachsel, H.; Doerksen, R. J.; Dreuw, A.; Dunietz, B. D.; Dutoi, A. D.; Furlani, T. R.; Gwaltney, S. R.; Heyden, A.; Hirata, S.; Hsu, C. P.; Kedziora, G.; Khalliulin, R. Z.; Klunzinger, P.; Lee, A. M.; Lee, M. S.; Liang, W. Z.; Lotan, I.; Nair, N.; Peters, B.; Proynov, E. I.; Pieniazek, P. A.; Rhee, Y. M.; Ritchie, J.; Rosta, E.; Sherrill, C. D.; Simmonett, A. C.; Subotnik, J. E.; Woodcock, H. L., III; Zhang, W.; Bell, A. T.; Chakraborty, A. K.; Chipman, D. M.; Keil, F. J.; Warshel, A.; Hehre, W. J.; Schaefer, H. F., III; Kong, J.; Krylov, A. I.; Gill, P. M. W.; Head-Gordon, M. Phys. Chem. Chem. Phys. 2006, 8 (27), 3172. doi:10.1039/ b517914a. PMID:16902710.

\section{Appendix}

Table A1 contains the fundamental integrals ${ }^{A l}$ (in the Coulomb metric) that are required to form $\gamma$ in single-center systems, such as atoms.

In the final expression, the integration limits are

$$
\text { [A1] } X=\frac{a}{\sqrt{a^{2}+b^{2}}} \frac{d}{\sqrt{b^{2}+d^{2}}}
$$

Table A1. Integrals required to form the $\gamma$ diagnostic (eq. [4]) in atomic systems.

\begin{tabular}{llll}
\hline$\langle f \mid g\rangle$ & $\exp \left(-d^{2} r^{2}\right)$ & $-\frac{\nabla^{2}}{2} \exp \left(-d^{2} r^{2}\right)$ & $\frac{\operatorname{erf}(c r) \exp \left(-d^{2} r^{2}\right)}{r}$ \\
\hline $\exp \left(-b^{2} r^{2}\right)$ & $\frac{2 \pi^{5 / 2}}{b^{2} d^{2} \sqrt{b^{2}+d^{2}}}$ & $\frac{2 \pi^{5 / 2}}{\left(b^{2}+d^{2}\right)^{3 / 2}}$ & $\frac{4 \pi^{2}}{b^{3} d} \tan ^{-1}\left[\frac{b}{d} \frac{c}{\sqrt{b^{2}+c^{2}+d^{2}}}\right]$ \\
$-\frac{\nabla^{2}}{2} \exp \left(-b^{2} r^{2}\right)$ & $\frac{2 \pi^{5 / 2}}{\left(b^{2}+d^{2}\right)^{3 / 2}}$ & $\frac{6 \pi^{5 / 2} b^{2} d^{2}}{\left(b^{2}+d^{2}\right)^{5 / 2}}$ & $\frac{4 \pi^{2}}{b^{2}+d^{2}} \frac{c}{\sqrt{b^{2}+c^{2}+d^{2}}}$ \\
$\frac{\operatorname{erf}(a r)}{r} \exp \left(-b^{2} r^{2}\right)$ & $\frac{4 \pi^{2}}{b d^{3}} \tan ^{-1}\left[\frac{d}{b} \frac{a}{\sqrt{a^{2}+b^{2}+d^{2}}}\right]$ & $\frac{4 \pi^{2}}{b^{2}+d^{2}} \frac{a}{\sqrt{a^{2}+b^{2}+d^{2}}}$ & $\left(\frac{4 \pi}{b d}\right)^{3 / 2} \sqrt{\frac{b^{2}+d^{2}}{b d}} \int_{0}^{X} \int_{0}^{Y} \frac{\mathrm{d} x \mathrm{~d} y}{\sqrt{1-x^{2}-y^{2}}}$ \\
\hline
\end{tabular}


[A2] $\quad Y=\frac{b}{\sqrt{b^{2}+d^{2}}} \frac{c}{\sqrt{c^{2}+d^{2}}}$

The double integral is elementary and can be evaluated in terms of arctangents but the resulting expression is messy.

\section{Reference}

(A1) Gill, P. M. W.; Johnson, B. G.; Pople, J. A. Int. J. Quantum Chem. 1991, 40 (6), 745. doi:10.1002/qua.560400604. 